10

\title{
Акустооптические модуляторы с расширенной частотной полосой для волоконно-оптических линий
}

\author{
(C) В.М. Епихин, А.В. Рябинин \\ Всероссийский научно-исследовательский институт фризико-технических и радиотехнических измерений, \\ 141570 Менделеево, Московская обл., Россия \\ e-mail: epikvm@mail.ru
}

Поступило в Редакцию 30 ноября 2020 г.

В окончательной редакции 21 декабря 2020 г.

Принято к публикации 23 декабря 2020 г.

Разработан и изготовлен модулятор-частотосдвигатель с одномодовыми волоконными световодами на длину волны излучения $1064 \mathrm{~nm}$. Световой пучок фокусировался в центре звукового столба. Время переключения модулятора $\simeq 18 \mathrm{~ns}$. Режим работы: импульсный, непрерывный. Общие оптические потери на центральной частоте $-3.2 \mathrm{~dB}$. Получено выражение для частотной полосы приема модулятора. Оценки дают хорошее согласие с данными эксперимента $\simeq 40 \mathrm{MHz}$. Показано, что использование схемы с фокусированным пучком дает возможность реализовать модулятор с минимальным временем переключения $\simeq 2-3$ ns и частотной полосой приема $\simeq 200-300 \mathrm{MHz}$.

Ключевые слова: акустооптические модуляторы, одномодовые волоконные световоды, время переключения, частотная полоса модулятора, частотная полоса переключения, частотная полоса согласования излучателя акустической волны, частотная полоса приема, ширина частотной аппаратной функции.

DOI: $10.21883 /$ JTF.2021.06.50874.330-20

\section{Введение}

Акустооптические устройства с одномодовыми волоконными световодами активно используются в оптических системах, содержащих волоконные линии, для коммутации оптических каналов связи (дефлекторы); изменения спектрального состава (фильтры), модуляции частоты и интенсивности оптических сигналов (модуляторы-частотосдвигатели) [1-3]. Акустооптические модуляторы-частотосдвигатели с одномодовыми волоконными выводами для краткости далее будем называть волоконными (АОМВ).

В [3] сообщается о создании АОМВ с коллимированным световым пучком, малыми оптическими потерями $\sigma<2 \mathrm{~dB}$ и полосой рабочих частот $\Delta f \approx \Delta f_{r} \sim 10 \mathrm{MHz}$, где $\Delta f_{r}$ - полоса приема, обусловленная уменьшением пространственной апертуры дифрагировавшего пучка с изменением частоты $f$ при его введении в приемный волоконно-оптический световод (ВОС). В качестве развития этих исследований представляют интерес АОМВ с расширенной частотной полосой и малым временем переключения.

Целью настоящей работы являлось создание АОМВ со световым пучком, фокусированным в центре звукового столба, и его экспериментальное исследование, а также определение механизма формирования рабочей полосы частот и оценка возможности реализации АОМВ с минимальным временем переключения $\tau_{\min } \simeq 2-3 \mathrm{~ns}$, значение которого ограничено напряжением пробоя излучателя ультразвуковой волны (УЗВ) [3].

\section{1. Время переключения и частотная полоса АОМВ с фокусированным световым пучком}

На рис. 1, а показана оптическая схема АОМВ с малым временем переключения. Микрообъектив с фокусирующей асферической линзой (далее для краткости фокусатор) $\Phi_{1}$ обеспечивает сходящийся гауссов пучок с перетяжкой в центре звукового столба акустооптической ячейки (AОC), причем ось падающего пучка $O$ направлена под углом Брэгга $\theta_{\mathrm{B}}$ к фронту УЗВ. Фокусатор $\Phi_{2}$, идентичный $\Phi_{1}$, предназначен для приема и передачи дифрагировавшего пучка в ВОС с минимальными потерями. Длина звукового столба вдоль светового пучка $L$ не должна превышать рэлеевской длины пучка $Z_{R}$ [4]: $L \leq Z_{R}$, тогда диаметр пучка принимается равным диаметру перетяжки $2 w$. Для получения максимальной интенсивности дифрагировавшего пучка при минимальной мощности УЗВ ширина звукового столба $H$ в ближней зоне излучателя УЗВ должна быть не меньше диаметра перетяжки $2 w: H \geq 2 w$. Быстродействие модулятора характеризуют временем переключения $\tau$, т.е. временем возрастания мощности дифрагировавшего пучка по уровню от 0.1 до 0.9 .

Практический интерес представляют АОМВ в режиме Брэгга при $a=\psi_{l g h} / \psi_{\text {snd }} \leq 1$, где $\psi_{l g h}$ и $\psi_{\text {snd }}-$ расходимости пучков света и звука соответственно, когда формируется единственный порядок дифракции, и максимально достижимое значение эффективности дифракции $\gtrsim 0.8$ [5]. В этом случае минимальное время переключения пропорционально времени пересечения 


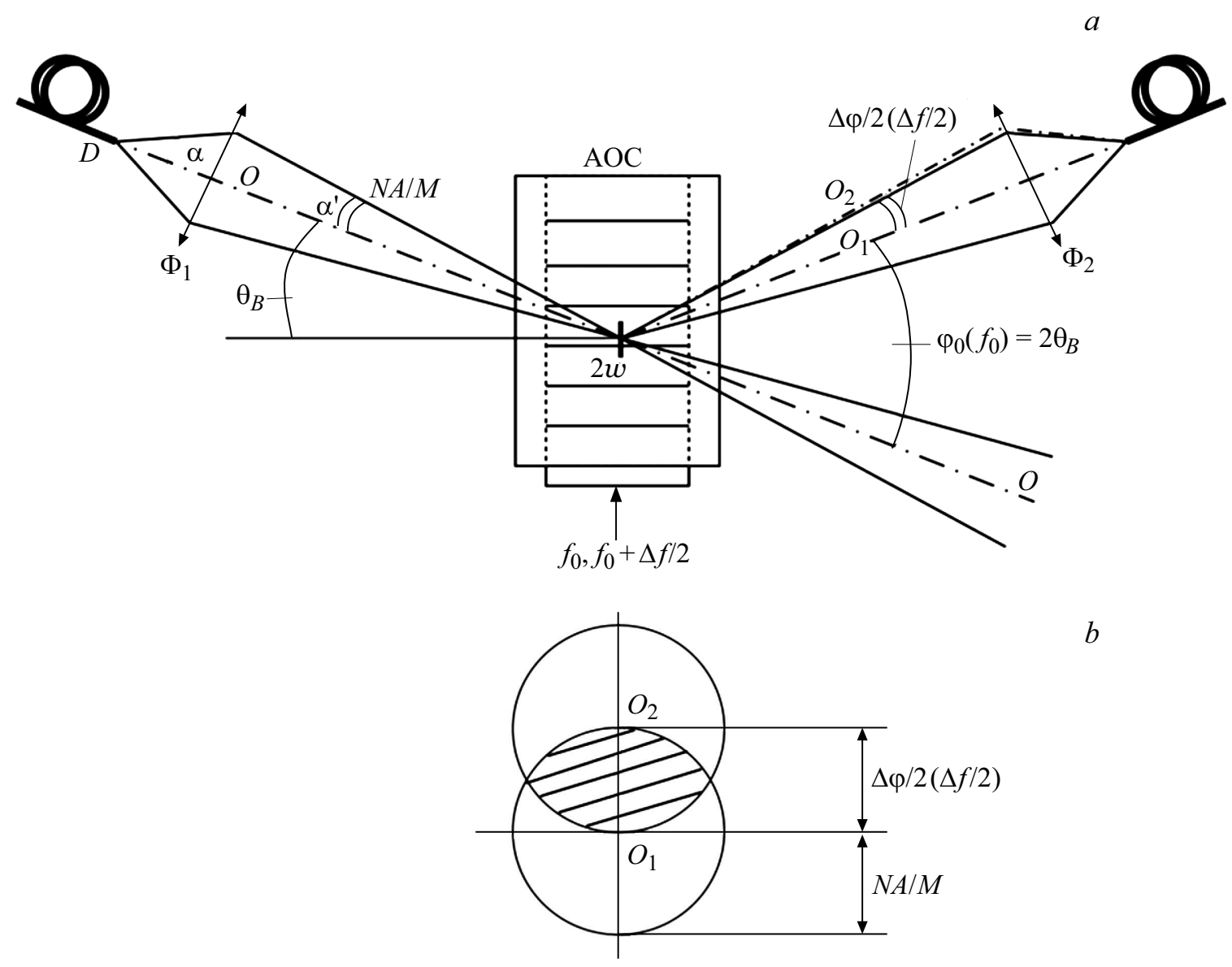

Рис. 1. $a-$ оптическая схема АОМВ с фокусировкой светового пучка; $b-$ расположение оптических осей $O_{1}$ и $O_{2}$ и угловых полей дифрагировавшего пучка при значениях частоты управляющего сигнала $f_{0}$ и $f_{0}+N A V /(M \lambda)$.

перетяжки светового гауссова пучка волновым фронтом У3В $[5,6]$ :

$$
\tau_{\text {cr }}=0.64(2 w / V)
$$

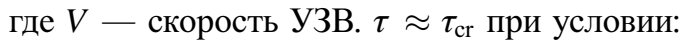

$$
\left(\Delta f_{m}, \Delta f_{a f}, \Delta f_{r}\right) \gtrsim \Delta f_{\mathrm{cr}} \simeq 0.5 / \tau_{\mathrm{cr}}
$$

где $\Delta f_{\text {cr }}$ - частотная полоса переключения по уровню $-3 \mathrm{~dB}$ (полоса модулирующих частот [5]), а в скобках перечислены все полосовые характеристики реального АОМВ: $\Delta f_{m}$ - частотная полоса согласования излучателя УЗВ с ВЧ-трактом $(50 \Omega)$, в пределах которой мощность управляющего сигнала от ВЧ-генератора передается в излучатель УЗВ с потерями $\leq 3 \mathrm{~dB}[6,7]$; $\Delta f_{a f}$ - ширина частотной аппаратной функции (полоса частот АО-взаимодействия), обратно пропорциональная длине АО-взаимодействия, в пределах которой эффективность дифракции составляет не менее 0.5 от максимального значения $[7,8] ; \Delta f_{r}$ - частотная полоса приема, в пределах которой мощность дифрагировавшего светового пучка передается в приемный ВОК с потерями $\leq 3 \mathrm{~dB}[3]$.
Если неравенство (2) не выполняется, $\tau>\tau_{\text {cr. }}$ Грубую оценку $\tau$ можно сделать по формуле:

$$
\tau \approx 0.5 / \Delta f, \quad \Delta f=\min \left(\Delta f_{m}, \Delta f_{a f}, \Delta f_{r}, \Delta f_{\text {cr }}\right),
$$

где $\Delta f$ - частотная полоса реального АОМВ в режиме формирователя временного фронта интенсивности дифрагировавшего света, поскольку коэффициент передачи системы равен произведению коэффициентов передачи ее составных частей, а длительность фронта импульса $\tau$ и полоса частот гармоник $\Delta f$, необходимая для его формирования, связаны соотношением $\tau \Delta f \simeq 0.5$ [8].

AOMB может также использоваться в режиме стационарного частотосдвигателя для получения сдвига частоты дифрагировавшего света в пределах рабочей полосы $\Delta f=\left(f_{0}+\Delta f / 2\right)-\left(f_{0}-\Delta f / 2\right)[9]$. В этом случае $\Delta f_{\text {cr }}$ не является ограничивающим фактором, и справедливо соотношение

$$
\Delta f=\min \left(\Delta f_{m}, \Delta f_{a f}, \Delta f_{r}\right) .
$$

Для классического АОМ со свободными световыми пучками отсутствуют элементы с узким угловым полем (кроме самой АОЯ), поэтому $\Delta f_{r}$ также не является ограничивающим фактором, и (4) переходит в $\Delta f=\min \left(\Delta f_{m}, \Delta f_{a f}\right)$. 
Таблица 1. Расчетные параметры АОМВ

\begin{tabular}{c|c|c|c|c|c|c|c|c|c|c|c}
\hline АОМВ & $\begin{array}{c}\text { Длина волны } \lambda, \\
\mathrm{nm}\end{array}$ & $\begin{array}{c}\text { Апертура } H, \\
\mu \mathrm{m}\end{array}$ & $\begin{array}{c}\text { Частота } f_{0}, \\
\mathrm{MHz}\end{array}$ & $a$ & $\begin{array}{c}\tau, \\
\mathrm{ns}\end{array}$ & $\begin{array}{c}\Delta f_{\text {cr calc }}^{\text {calc }} \\
\mathrm{MHz}\end{array}$ & $\begin{array}{c}\Delta f_{r}^{\text {calc }} \\
\mathrm{MHz}\end{array}$ & $\begin{array}{c}\Delta f_{\text {af calc }}^{\text {calc }} \\
\mathrm{MHz}\end{array}$ & $Q / 4 \pi$ & $T_{M}$ & $\begin{array}{c}P_{M}^{a}, \\
\mathrm{~W}\end{array}$ \\
\hline Фотон-6203 & 1064 & 120 & 200 & 1 & 18 & 28 & 36 & 95 & 1.9 & 0.8 & 0.65
\end{tabular}

Рассмотрим более подробно важную характеристику АОМВ - частотную полосу приема $\Delta f_{r}$, поскольку этот параметр часто является определяющим при формировании $\Delta f$ в (3). На рис. 1, $a$ показана упрощенная модель АО-дифракции в АОМВ. Здесь источником дифрагировавшего излучения служит перетяжка падающего пучка диаметром $2 w$ в центре АОЯ, которая является увеличенным изображением перетяжки исходного пучка на торце ВОС фокусатора $\Phi_{1}$ с диаметром $D$, равным диаметру модового пятна $[4,10]$. Увеличение оптической системы $M=2 w / D$. Изображение перетяжки $2 w$ симметрично переносится на торец приемного ВОС с помощью $\Phi_{2}$. Угол расходимости светового пучка (по уровню $1 / e^{2}$ ) до и после линзы фокусатора равен $N A$ и $N A / M$ соответственно, где $N A-$ эффективная числовая апертура ВОС [10].

При частоте управляющего сигнала $f_{0}$ угол $\varphi_{0}$ между нулевым (ось $O$ ) и первым (ось $O_{1}$ ) порядками дифракции равен

$$
\varphi_{0}\left(f_{0}\right)=2 \theta_{\mathrm{B}} \approx \lambda f / V,
$$

а ось $O_{1}$ и угловое поле дифрагировавшего пучка совпадают с осью и угловым полем приемного ВОС. При перестройке частоты $f$ от $f_{0}$ до $\left(f_{0}+\Delta f / 2\right)$ расположение перетяжек остается прежним, однако угловая структура поля изменяется, и ось пучка $O_{1}$ занимает положение $O_{2}$ под углом $\Delta \varphi / 2$. Связь между $\Delta f$ и $\Delta \varphi$ получается дифференцированием обеих частей формулы (5), и ее можно записать следующим образом:

$$
\Delta \varphi / 2=\lambda \Delta f /(2 V) .
$$

При угле $\Delta \varphi / 2=N A / M$ угловые поля дифрагировавших пучков с осями $O_{1}$ и $O_{2}$ будут иметь вид, паказанный на рис. $1, b$. Окружности изображают границы углового распределения по уровню интенсивности $1 / e^{2}$. Для элементарных оценок примем: 1) угловые поля ограничены указанными окружностями, а распределение интенсивности пучка в пределах окружности равномерное; 2) угловые поля дифрагировавших пучков и приемного ВОС идентичны; 3) оптическая система идеально сьюстирована для $f=f_{0}$. Тогда доля интенсивности света, переданная в ВОС $\Phi_{2}$ приблизительно равна отношению заштрихованной площади к площади круга, и она составляет $0.39(\simeq-4 \mathrm{~dB})$ [3]. В результате получаем формулу для частотной полосы приема по указанному уровню:

$$
\Delta f_{r} \simeq 2 N A V /(M \lambda)
$$

Как и в случае коллимированного пучка, полоса приема пропорциональна $V$, поэтому целесообразно использовать изотропную дифракцию на продольной УЗВ, где скорость $V$ значительно выше [3]. Итак, механизм формирования полосы приема модулятора заключается в ограничении апертуры дифрагировавшего пучка при вводе его в приемный ВОС. При этом для АОМВ с коллиматорами ограничивается линейная апертура, а для $\mathrm{AOMB}$ с фокусаторами - угловая апертура.

В качестве примера оценим относительное увеличение полосы $k$ для $\lambda=1064 \mathrm{~nm}$ при переходе от коллимированного пучка к фокусированному при прочих равных условиях:

$$
\begin{aligned}
k= & f_{\text {foc }} / f_{\text {col }}=\left(\frac{2 N A V}{M \lambda}\right) /\left(\frac{D V}{F \lambda}\right) \\
& =2 N A F /(M D)=N A F / w .
\end{aligned}
$$

Подставляя $N A=0.11$ [10], $F=4 \mathrm{~mm}[3], w=H / 2=$ $=0.1-0.01 \mathrm{~mm}$, получаем $k \approx 4-40$. Следовательно, схема с фокусированным пучком дает радикальное расширение полосы приема.

\section{2. Расчетные параметры и конструкция АОМВ}

По аналогии с [3] в настоящей работе использовалась изотропная дифракция на продольной УЗВ, распространяющейся по оси $Z$ в кристалле $\mathrm{TeO}_{2}$ со скоростью $V=4260 \mathrm{~m} / \mathrm{s}$. Ширина звукового столба (активная апертура) $H=120 \mu \mathrm{m}$. При $H=2 w$ получаем время переключения $\tau \simeq 18 \mathrm{~ns}$, что соответствует частотной полосе переключения $\Delta f_{\text {cr }} \simeq 28 \mathrm{MHz}$. Поскольку для близких значений акустических импедансов материалов АО-кристалла и пьезопреобразователя (ПП) $\Delta f_{m} \sim f_{0}$ и может достигать октавы [8], для облегчения согласования ПП с ВЧ-трактом и обеспечения неравенства $\Delta f_{m}>\Delta f_{\text {cr }}$ была выбрана центральная частота $f_{0}=200 \mathrm{MHz}$. Длина ПП вдоль пучка света выбиралась из равенства $a=1$. В табл. 1 представлены расчетные параметры АОМВ Фотон-6203.

Частотные полосовые характеристики по уровню $-3 \mathrm{~dB}$ вычислялись по формулам: $\Delta f_{r}^{\text {calc }}=1.6 N A V /(M \lambda), \quad$ где $\quad N A=0.11, \quad M=2 w / D=$ $=120 \mu \mathrm{m} / 6.2 \mu \mathrm{m} \simeq 19.4$ [10], $\Delta f_{a f}^{\text {calc }}=1.77 V^{2} n /\left(f_{0} L \lambda\right)[8]$, где $n \simeq 2.3$ - показатель преломления $\mathrm{TeO}_{2}$, вычисленный по формулам Селмейера, экстраполированным в ИК-диапазон [11], $Q$ - параметр Кляйна-Кука [12], $T_{M}$ - максимально достижимая эффективность дифракции $[5], P_{M}^{a}-$ мощность УЗВ, необходимая для получения $T_{M}[6]$. 
Таблица 2. Экспериментальные параметры АОМВ

\begin{tabular}{c|c|c|c|c|c|c|c}
\hline АОМВ & $\begin{array}{c}\text { Длина волны } \\
\lambda, \mathrm{nm}\end{array}$ & $\begin{array}{c}\text { Общие оптические } \\
\text { потери } \sigma, \mathrm{dB}\end{array}$ & $\begin{array}{c}\text { Контраст } \\
\text { модуляции } K, \mathrm{~dB}\end{array}$ & $\begin{array}{c}\Delta f_{m}^{\mathrm{exp}}, \\
\mathrm{MHz}\end{array}$ & $\begin{array}{c}\Delta f_{a f}^{\text {exp }} \\
\mathrm{MHz}\end{array}$ & $\begin{array}{c}\Delta f_{r}^{\text {exp }} \\
\mathrm{MHz}\end{array}$ & $\begin{array}{c}P_{M}^{H F}, \\
\mathrm{~W}\end{array}$ \\
\hline Фотон-6203 & $1064 \pm 40$ & -3.2 & 63 & 80 & 97 & 41 & 0.95
\end{tabular}

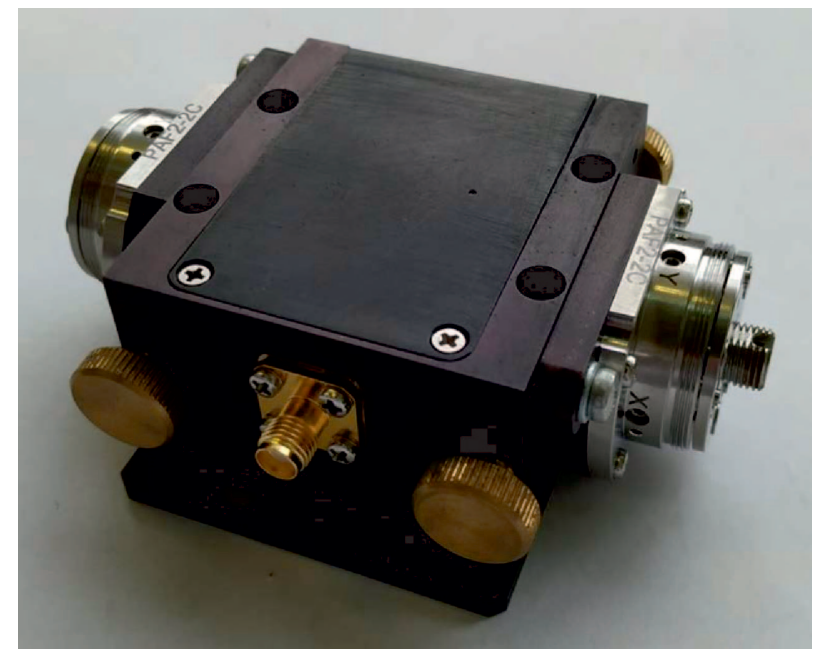

Рис. 2. Фото АОМВ Фотон-6203 с фокусировкой светового пучка.

На рис. 2 представлено фото АОМВ Фотон-6202. АОМВ состоит из корпуса размерами $95 \times 70 \times 37 \mathrm{~mm}$, внутри которого находятся АОЯ, устройство согласования импедансов ПП и ВЧ-тракта $(50 \Omega)$ и асферические линзовые входной и выходной настраиваемые объективы Fiberport PAF2-2C с тремя линейными и тремя угловыми степенями свободы, имеющие оптическими разъемы FC/APC [13]. Объективы служили фокусаторами с изменяемыми расстояниями $a$ и $a^{\prime}$.

\section{3. Экспериментальные результаты и их обсуждение}

В табл. 2 приведены результаты экспериментального исследования изготовленного АОМВ Фотон-6203 с фокусированным пучком.

Для длины волны излучения указан спектральный диапазон, в котором коэффициент отражения не превышает $0.2 \%$. Определение $\sigma$ и $K$ дано в [3], их значения указаны в табл. 2 для $f_{0}=200 \mathrm{MHz}$. Полоса согласования ПП $\Delta f_{m}^{\exp }$ измерялась по уровню КСВН $\leq 1.3$, что соответствует уровню отраженной ВЧ мощности $\leq 2 \%$. Ширина частотной аппаратной функции $\Delta f_{a f}^{\exp }$ измерялась при установленном входном фокусаторе $\Phi_{1}$ без приемного фокусатора $\Phi_{2}$. В этом случае непосредственно за АОЯ устанавливался широкоаппертурный фотоприемник с угловой апертурой $\Delta \alpha \gg 2 N A / M \simeq 0.7^{\circ}$. Приемная полоса $\Delta f_{r}^{\text {exp }}$ измерялась при установленных фокусаторах $\Phi_{1}$ и $\Phi_{2} . P_{M}^{H F}-$ мощность управляющего ВЧ-сигнала, при которой значение $\sigma$ минимально. Источником излучения служил лазер LCS-T-12, а приемником - измеритель мощности РМ20С. Для соединения источника и приемника с АОМВ использовались отрезки BOC P3-980A-FC-1 длиной $1 \mathrm{~m}$.

АОМВ Фотон-6203 был испытан как в импульсном, так и в непрерывном режимах без изменения его параметров. Это согласуется с данными [9], где для АОМ Фотон-3208 с близкими размерами ПП была экспериментально определена максимальная средняя по времени мощность УЗВ без деструкции ПП: $\left\langle P_{M}^{a}\right\rangle \simeq 1 \mathrm{~W}$.

Из полученных результатов можно сделать следующие выводы:

1. Неравенство (2), как необходимое и достаточное условие получения расчетного времени переключения $\tau \simeq 18 \mathrm{~ns}$, в данном случае выполняется.

2. Несмотря на упрощающие допущения, сделанные при выводе формулы (7) для $\Delta f_{r}$, расчетные и экспериментальные данные находятся в хорошем согласии. Это относится к результатам, полученным как в [3], так и в настоящей работе.

3. При использовании АОМВ в качестве стационарного частотосдвигателя его рабочая полоса определяется частотной полосой приема $\Delta f \simeq \Delta f_{r} \simeq 40 \mathrm{MHz}$. На краях этой полосы общие оптические потери составляют $\sigma\left(f_{0} \pm \Delta f / 2\right) \simeq 3 \mathrm{~dB}+3.2 \mathrm{~dB}=6.2 \mathrm{~dB}$. Согласно данным табл. 2, значение полосы частот АО-взаимодействия $\Delta f_{a f}^{\text {exp }}$ значительно превышает $\Delta f_{r}$. Следовательно, основная доля потерь на краях рабочей полосы обусловлена потерями ввода дифрагировавшего светового пучка в приемный ВОС.

\section{4. Возможность реализации АОМВ с минимальным временем переключения}

В работе [3] сделаны оценки минимальных значений времени переключения $\mathrm{AOMB} \tau_{\min }$, исходя из максимального значения плотности акустической мощности, ограниченной электрическим пробоем ПП. Интересно рассмотреть возможность практической реализации данных [3] с учетом результатов, полученных в настоящей работе.

В табл. 3 сведены параметры, вычисленные по формулам (1)-(5), с учетом [3] и исходя из значений пороговой плотности акустической мощности без деструкции ПП в импульсном режиме $\rho_{\mathrm{imp}} \approx 360 \cdot 10^{6} \mathrm{~W} / \mathrm{m}^{2}$. 
Таблица 3. Расчетные параметры АОМВ с минимальным временем переключения

\begin{tabular}{c|c|c|c|c|c|c|c|c|c|c}
\hline $\begin{array}{c}\lambda, \\
\mathrm{nm}\end{array}$ & $\begin{array}{c}L, \\
\mu \mathrm{m}\end{array}$ & $\begin{array}{c}H, \\
\mu \mathrm{m}\end{array}$ & $\begin{array}{c}f_{0}, \\
\mathrm{MHz}\end{array}$ & $\begin{array}{c}\tau_{\min }, \\
\mathrm{ns}\end{array}$ & $\begin{array}{c}\Delta f_{\mathrm{cr}}, \\
\mathrm{MHz}\end{array}$ & $\begin{array}{c}\Delta f_{m}, \\
\mathrm{MHz}\end{array}$ & $\begin{array}{c}\Delta f_{r}, \\
\mathrm{MHz}\end{array}$ & $\begin{array}{c}\Delta f_{a f}, \\
\mathrm{MHz}\end{array}$ & $\begin{array}{c}P_{a}, \\
\mathrm{~W}\end{array}$ & $m$ \\
\hline 1064 & 225 & 14.6 & 455 & 2.2 & 227 & 303 & 300 & 650 & 1.2 & 1.2 \\
\hline 1550 & 323 & 21.2 & 313 & 3.2 & 156 & 209 & 205 & 451 & 2.5 & 2.5
\end{tabular}

Полоса согласования ПП полагалась равной октаве, т.е. $\Delta f_{m}=2 f_{0} / 3$. Осуществить такое значение возможно вследствие близости значений акустических импедансов ПП $\left(\mathrm{LiNbO}_{3} y+36^{\circ}\right.$-среза $)$ и светозвукопровода $\left(\mathrm{TeO}_{2} z\right.$-среза): $34.3 \cdot 10^{6} \mathrm{kgm}^{-2} \cdot \mathrm{s}^{-1}$ и $25.2 \cdot 10^{6} \mathrm{kgm}^{-2} \cdot \mathrm{s}^{-1}$ соответственно [8]. В последнем столбце табл. 3 приведены минимальные значения скважности импульсной последовательности управляющего ВЧ сигнала $m$, при которой средняя по времени мощность $\left\langle P_{a}\right\rangle$ не превышает „безопасного“ для теплового режима ПП уровня $1 \mathrm{~W}$.

Из табл. 3 видно, что неравенство (2) выполняется для обеих длин волн излучения 1064 и $1550 \mathrm{~nm}$. Это позволяет утверждать, что схема с фокусированным пучком дает возможность реализовать АОМВ с $\tau$, близким к предельным значениям $\tau_{\min }$, ограниченным напряжением пробоя используемого ПП.

В [14-17] подробно представлена информация ведущих фирм-производителей об акустооптических прибоpax c оптоволоконными выводами. Из анализа этих данных следует: 1) отечественные производители отсутствуют; 2) разработанный АОМВ Фотон-6203 не уступает аналогам фирм-лидеров по основным параметрам: времени переключения, частоте модуляции, мощности управляющего ВЧ-сигнала, оптическим потерям и режимам работы; 3) рассмотренные в настоящей работе АОМВ с минимальнным временем переключения $\simeq 2-3 \mathrm{~ns}$ не имеют аналогов.

Разработанное устройство может использоваться для увеличения диапазона амплитудной и частотной модуляции лазеров с оптоволоконными выводами; в качестве селектора импульсов в волоконных системах с высокой частотой повторения для увеличение диапазона частоты повторения; при создании транспортируемых оптических и микроволновых стандартов частоты [9] для уменьшения их габаритов; в волоконно-оптических датчиках и дальномерах для увеличения пространственного разрешения [18].

\section{Выводы}

1. Рассмотрена и экспериментально исследована схема $\mathrm{AOMB}$ со световым пучком, сфокусированным в центре звукового столба АОЯ. Определены условия, необходимые и достаточные для получения времени переключения, определяемого размером перетяжки светового пучка и скоростью акустической волны.
2. Получено выражение для частотной полосы приема АОМВ. Показано, что схема с фокусировкой пучка дает радикальное расширение частотной полосы приема.

3. Изготовлен АОМВ с фокусировкой пучка и временем переключения $\simeq 18 \mathrm{~ns}$ и исследованы его экспериментальные параметры. Расчетное и измеренное значения частотной полосы приема находятся в хорошем согласии.

4. Сделаны оценки параметров АОМВ с минимально возможным временем переключения, определяемым электрическим пробоем пьезопреобразователя. Показано, что схема с фокусированным световым пучком позволяет реализовать модуляторы с временем переключения, близким к минимальным значениям $\tau_{\min } \simeq 2-3 \mathrm{~ns}$.

\section{Конфликт интересов}

Авторы заявляют, что у них нет конфликта интересов.

\section{Список литературы}

[1] В.Я. Молчанов, Ю.И. Китаев, А.И. Колесников, В.Н. Нарвер, А.3. Розенштейн, Н.П. Солодовников, К.Г. Шаповаленко. Теория и практика современной акустооптики (Изд. Дом МИСиС, М., 2015), с. 77.

[2] С.Н. Антонов. ЖТФ, 89 (2), 274 (2019).

[3] В.М. Епихин, П.В. Карнаушкин. Квант. электрон., 50 (10), 962 (2020).

[4] Ю. Айхлер, Г.И. Айхлер. Лазеры. Исполнение, управление, применение (Техносфера, М., 2012), с. 240.

[5] D. Maydan. IEEE J., QE-6 (1), 15 (1970).

[6] Л.Н. Магдич, В.Я. Молчанов. Акустооптические устройства и их применение (Сов. радио, М., 1978), с. 31.

[7] В.И. Балакший, В.Н. Парыгин, Л.Е. Чирков. Физические основы акустооптики (Радио и связь, М., 1985), с. 45.

[8] Э. Дьелесан, Д. Руайе. Упругие волны в твердых телах. Применение для обработки сигналов (Наука, М., 1982), c. 338.

[9] В.М. Епихин, В.Н. Барышев, С.Н. Слюсарев, А.В. Апрелев, И.Ю. Блинов. Квант. электрони., 49 (9), 857 (2019).

[10] OZ Optics Ltd. [Электронный ресурс] Режим доступа: www.ozoptics.com

[11] N. Uchida. Phys. Rev. B, 4 (10), 3736 (1971).

[12] W.R. Klein, D.D. Cook. IEEE Trans., SU-14 (3), 123 (1967).

[13] Thorlabs, Inc. [Электронный pecypc] Режим доступа: www.thorlabs.com

[14] Акустооптические модуляторы с оптоволокном [Электронный ресурс] Режим доступа: https://sphotonics.ru/ catalog/sm-aom/

[15] Isomet Corporation [Электронный ресурс] Режим доступа: https://www.isomet.com

[16] Brimrose Corporation of America [Электронный pecypc] Режим доступа: https://www.brimrose.com

[17] G\& H [Электронный ресурс] Режим доступа: https://www.gandh.com

[18] Т.О. Лукашова, О.Е. Наний, С.П. Никитин, В.Н. Трещиков. Квант. электрон., 50 (9), 882 (2020). 